\title{
Crystal structure of $\boldsymbol{P}, \boldsymbol{P}$-diphenyl- $\boldsymbol{N}$-ethyl-selenophosphinylthioformamide, $\mathrm{C}_{15} \mathrm{H}_{16}$ NPSSe
}

\author{
R. Kramolowsky, J. Sawluk
}

Universität Hamburg. Institut für Anorganische und Angewandte Chemie, D-20146 Hamburg. Germany

G. Siasios and E. R. T. Tiekink

The University of Adelaide, Department of Chemistry, South Australia 5005, Australia

Received December 2, 1996, CSD-No. 402729

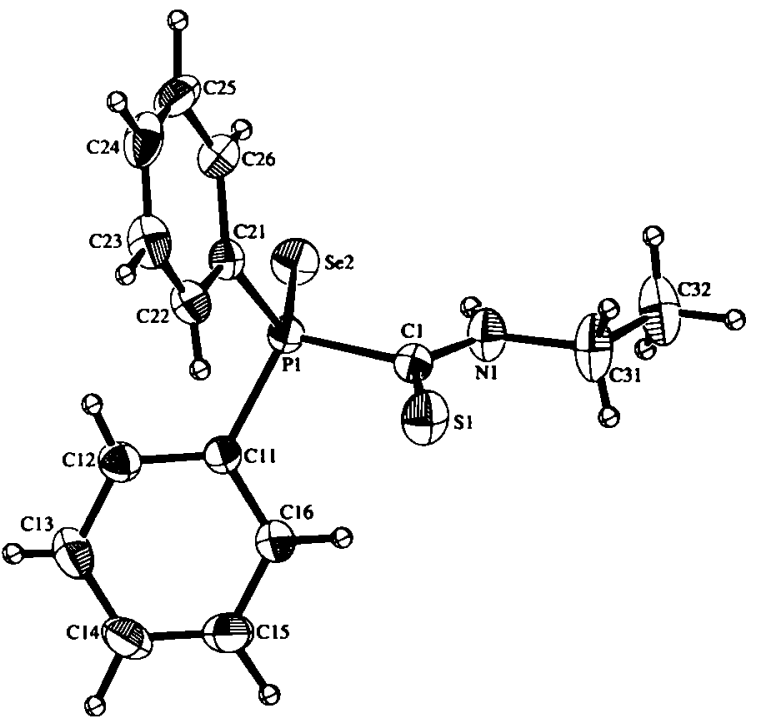

Source of material: Prepared using the literature method (see ref. 1); mp $371 \mathrm{~K}$.

The conformation about the $\mathrm{C}(1)-\mathrm{N}(1)$ bond is $Z$ and the central chromophore is planar as seen in the values of the $\operatorname{Se}(2) / \mathrm{P}(1)$ / $\mathrm{C}(1) / \mathrm{S}(1)$ and $\mathrm{Se}(2) / \mathrm{P}(1) / \mathrm{C}(1) / \mathrm{N}(1)$ torsion angles of $178.8(6)^{\circ}$ and $-0.3(4)^{\circ}$, respectively. The derived interatomic parameters are equal within experimental error to those of the $N$-methyl analogue (see ref. 2), indicating that the substitution of a methyl group for an ethyl has little effect on the electronic structures of the compounds.

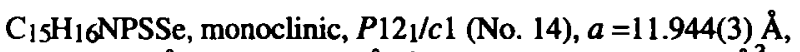
$b=8.999(7) \AA, c=14.882(3) \AA, \beta=100.71(2)^{\circ}, V=1571.7 \AA^{3}, Z=4$, $R(F)=0.036, R_{W}(F)=0.034$.
Table 1. Parameters used for the $X$-ray data collection

$\begin{array}{ll}\text { Crystal: } & \text { yellow block, size } 0.19 \times 0.23 \times 0.24 \mathrm{~mm} \\ \text { Wavelength: } & \text { Mo } K_{\alpha} \text { radiation }(0.7107 \AA) \\ \mu: & 26.10 \mathrm{~cm}^{-1} \\ \text { Diffractometer: } & \text { AFC6R } \\ \text { Scan mode: } & \omega / 2 \theta \\ \text { T }_{\text {mearurement: }} & 293 \mathrm{~K} \\ 2 \theta_{\max }: & 55^{\circ} \\ \mathrm{N}(h k)_{\text {menique: }} & 1761 \\ \text { Criterion for } F_{\mathrm{o}}: & \mathrm{F}_{\mathrm{o}}>6 \sigma\left(F_{\mathrm{o}}\right) \\ \text { N(param })_{\text {refined: }} & 236 \\ \text { Program: } & \text { teXsan }\end{array}$

Table 2. Final atomic coordinates and displacement parameters (in $\AA^{2}$ )

\begin{tabular}{llllll}
\hline Atom & Site & $x$ & $y$ & $z$ & $U_{\text {iso }}$ \\
\hline H(1) & $4 e$ & $0.556(3)$ & $0.399(4)$ & $0.635(3)$ & $0.04(1)$ \\
H(12) & $4 e$ & $0.808(3)$ & $0.261(4)$ & $0.394(2)$ & $0.03(1)$ \\
$H(13)$ & $4 e$ & $0.883(4)$ & $0.406(6)$ & $0.299(3)$ & $0.08(1)$ \\
$H(14)$ & $4 e$ & $0.873(4)$ & $0.663(5)$ & $0.299(3)$ & $0.07(1)$ \\
$H(15)$ & $4 e$ & $0.778(4)$ & $0.767(5)$ & $0.407(3)$ & $0.07(1)$ \\
$H(16)$ & $4 e$ & $0.709(3)$ & $0.608(4)$ & $0.504(3)$ & $0.04(1)$ \\
$H(22)$ & $4 e$ & $0.930(4)$ & $0.244(5)$ & $0.573(3)$ & $0.05(1)$ \\
$H(23)$ & $4 e$ & $1.049(4)$ & $0.044(5)$ & $0.614(3)$ & $0.07(1)$ \\
$H(24)$ & $4 e$ & $0.972(4)$ & $-0.179(6)$ & $0.639(3)$ & $0.09(1)$ \\
$H(25)$ & $4 e$ & $0.767(3)$ & $-0.208(5)$ & $0.616(3)$ & $0.05(1)$ \\
$H(26)$ & $4 e$ & $0.651(3)$ & $0.004(4)$ & $0.562(3)$ & $0.06(1)$ \\
$H(31 a)$ & $4 e$ & $0.635(5)$ & $0.461(6)$ & $0.799(4)$ & $0.10(1)$ \\
$H(31 b)$ & $4 e$ & $0.614(6)$ & $0.612(8)$ & $0.742(5)$ & $0.162(8)$ \\
$H(32 a)$ & $4 e$ & $0.469(4)$ & $0.582(6)$ & $0.816(4)$ & $0.11(1)$ \\
$H(32 b)$ & $4 e$ & $0.441(5)$ & $0.579(7)$ & $0.711(4)$ & $0.12(1)$ \\
$H(32 c)$ & $4 e$ & $0.477(8)$ & $0.43(1)$ & $0.786(6)$ & $0.229(7)$
\end{tabular}

Table 3. Final atomic coordinates and displacement parameters (in $\AA^{2}$ )

\begin{tabular}{|c|c|c|c|c|c|c|c|c|c|c|}
\hline Atom & Site & $x$ & $y$ & $z$ & $U_{11}$ & $U_{22}$ & $U_{33}$ & $U_{12}$ & $U_{13}$ & $U_{23}$ \\
\hline $\operatorname{Se}(2)$ & $4 e$ & $0.52365(4)$ & $0.24677(6)$ & $0.48055(3)$ & $0.0362(2)$ & $0.0694(4)$ & $0.0668(3)$ & $-0.0088(3)$ & $-0.0009(2)$ & $-0.0074(3)$ \\
\hline $\mathbf{S}(1)$ & $4 e$ & $0.8292(1)$ & $0.4600(2)$ & $0.69682(9)$ & $0.0520(8)$ & $0.087(1)$ & $0.0619(8)$ & $-0.0098(7)$ & $-0.0024(6)$ & $-0.0211(8)$ \\
\hline$P(1)$ & $4 e$ & $0.69252(9)$ & $0.3011(1)$ & $0.53392(8)$ & $0.0331(6)$ & $0.0424(7)$ & $0.0421(7)$ & $-0.0025(5)$ & $0.0052(5)$ & $-0.0035(5)$ \\
\hline$N(1)$ & $4 e$ & $0.6091(4)$ & $0.4327(5)$ & $0.6665(3)$ & $0.047(3)$ & $0.080(3)$ & $0.052(3)$ & $0.001(2)$ & $0.008(2)$ & $-0.017(3)$ \\
\hline $\mathrm{C}(1)$ & $4 e$ & $0.7051(4)$ & $0.4083(5)$ & $0.6418(3)$ & $0.043(3)$ & $0.044(3)$ & $0.042(3)$ & $0.002(2)$ & $0.006(2)$ & $0.000(2)$ \\
\hline$C(11)$ & $4 e$ & $0.7563(3)$ & $0.4165(5)$ & $0.4602(3)$ & $0.036(2)$ & $0.044(3)$ & $0.039(3)$ & $-0.004(2)$ & $0.005(2)$ & $-0.003(2)$ \\
\hline$C(13)$ & $4 e$ & $0.8500(5)$ & $0.4461(8)$ & $0.3346(4)$ & $0.076(4)$ & $0.085(5)$ & $0.051(4)$ & $0.009(4)$ & $0.025(3)$ & $0.005(4)$ \\
\hline
\end{tabular}


Table 3. (Continued)

\begin{tabular}{|c|c|c|c|c|c|c|c|c|c|c|}
\hline Atom & Site & $x$ & $y$ & $z$ & $U_{11}$ & $U_{22}$ & $U_{33}$ & $U_{12}$ & $U_{13}$ & $U_{23}$ \\
\hline$C(14)$ & $4 e$ & $0.8413(5)$ & $0.5953(8)$ & $0.3401(4)$ & $0.084(4)$ & $0.082(5)$ & $0.057(4)$ & $-0.027(4)$ & $0.021(3)$ & $0.010(4)$ \\
\hline$C(15)$ & $4 e$ & $0.7888(5)$ & $0.6571(7)$ & $0.4038(4)$ & $0.106(5)$ & $0.054(4)$ & $0.068(4)$ & $-0.018(4)$ & $0.021(4)$ & $0.003(3)$ \\
\hline$C(16)$ & $4 e$ & $0.7461(5)$ & $0.5668(6)$ & $0.4631(4)$ & $0.075(4)$ & $0.055(4)$ & $0.053(3)$ & $-0.008(3)$ & $0.024(3)$ & $-0.006(3)$ \\
\hline$C(21)$ & $4 e$ & $0.7826(3)$ & $0.1440(5)$ & $0.5639(3)$ & $0.039(3)$ & $0.048(3)$ & $0.041(3)$ & $0.003(2)$ & $0.009(2)$ & $-0.003(2)$ \\
\hline$C(22)$ & $4 e$ & $0.8998(4)$ & $0.1550(6)$ & $0.5778(3)$ & $0.049(3)$ & $0.060(4)$ & $0.045(3)$ & $0.003(3)$ & $0.010(2)$ & $0.003(3)$ \\
\hline$C(23)$ & $4 e$ & $0.9657(5)$ & $0.0333(7)$ & $0.6036(3)$ & $0.057(4)$ & $0.081(5)$ & $0.055(3)$ & $0.027(3)$ & $0.009(3)$ & $0.01 /(3)$ \\
\hline$C(25)$ & $4 e$ & $0.8018(6)$ & $-0.1118(6)$ & $0.6018(4)$ & $0.095(5)$ & $0.036(3)$ & $0.067(4)$ & $0.003(3)$ & $0.009(3)$ & $-0.004(3)$ \\
\hline$C(26)$ & $4 e$ & $0.7342(5)$ & $0.0093(6)$ & $0.5753(3)$ & $0.059(3)$ & $0.049(3)$ & $0.057(3)$ & $0.001(3)$ & $0.009(3)$ & $-0.006(3)$ \\
\hline$C(31)$ & $4 e$ & $0.5966(6)$ & $0.5125(8)$ & $0.7489(4)$ & $0.079(4)$ & $0.121(6)$ & $0.058(4)$ & $0.016(4)$ & $0.015(3)$ & $-0.031(4)$ \\
\hline$C(32)$ & $4 e$ & $0.4770(6)$ & $0.5243(9)$ & $0.7584(5)$ & $0.095(5)$ & $0.145(7)$ & $0.061(5)$ & $0.042(5)$ & $0.026(4)$ & $-0.008(5)$ \\
\hline
\end{tabular}

Acknowledgments. G. S. was the holder of an Australian Postgraduate Research Award. The Australian Research Council is thanked for support.

\section{References}

1. Siasios, G.; Tiekink, E. R. T.: Crystal and molecular structures of $P, P$-diphenyl- $N$-methyl-phosphinylthioformamide and $P, P$-diphenyl- $N$-phenylselenophosphinylthioformamide. Z. Kristallogr. 207 (1993) 59-67.
2. Siasios, G.; Tiekink, E.R.T.: Crystal and molecular structures of $P, P$-diphenyl- $N$-methyl-selenophosphinylthioformanide, $P, P$-dicyclohexyl $N$ methyl-selenophosphinylthioformamide and $P, P$-dicyclohexyl- $N$-phenylselenophosphinylthioformamide. Z. Kristallogr. 210 (1995) 868-872.

3. teXsan. Single crystal structure analysis software. Version 1.6. Molecular Structure Corporation, The Woodlands, Texas, USA 1993. 